Journal of Applied Pharmaceutical Science Vol. 7 (01), pp. 120-128, January, 2017

Available online at http://www.japsonline.com

DOI: 10.7324/JAPS.2017.70116

ISSN 2231-3354 (cc) BY-NC-SA

\title{
In silico ADME/T and 3D QSAR analysis of KDR inhibitors
}

\author{
S. M. Zahid Hosen ${ }^{1}$, Raju Dash ${ }^{1}$, Mahmuda Khatun ${ }^{1}$, Rasheda Akter ${ }^{1}$, Md. Habibur Rahman Bhuiyan ${ }^{2}$, Md. Rezaul \\ Karim $^{3}$, Nusrat Jahan Mouri ${ }^{4}$, Forkan Ahamed ${ }^{5}$, Kazi Saiful Islam ${ }^{6}$, Sadia Afrin ${ }^{7}$ \\ ${ }^{1}$ Molecular Modeling \& Drug Design Laboratory (MMDDL), Pharmacology Research Division, Bangladesh Council of Scientific \& Industrial Research \\ (BCSIR), Chittagong-4220, Bangladesh. ${ }^{2}$ Industrial Microbiology Research Division, Bangladesh Council of Scientific \& Industrial Research (BCSIR), \\ Chittagong-4220, Bangladesh. ${ }^{3}$ Institute of Food Science \& Technology (IFST), BCSIR, Dhaka. ${ }^{4}$ Industrial Botany Research Division, Bangladesh Council \\ of Scientific \& Industrial Research (BCSIR), Chittagong-4220, Bangladesh. ${ }^{5}$ Department of Microbiology, Faculty of Biological Science, University of \\ Chittagong, Chittagong, Bangladesh. ${ }^{6}$ Charles Perkins Center-University of Sydney, NSW 2050, Australia. ${ }^{7}$ Department of Nutritional Biochemistry, \\ ICDDR, B, Dhaka 1212, Bangladesh.
}

\begin{tabular}{l} 
ARTICLE INFO \\
\hline Article history: \\
Received on: 16/08/2016 \\
Revised on: 17/09/2016 \\
Accepted on: 30/09/2016 \\
Available online: 31/01/2017 \\
\hline Key words: \\
N-4- \\
chlorophenylnaphthamides, \\
QSAR, QSTR, HUMO, \\
LUMO, LD ${ }_{50}$, KDR
\end{tabular}

\begin{abstract}
Tyrosine kinases (KDR) have been considered as a potential targets for the design of new anticancer agents. Recently, a series of N-4-chlorophenylnaphthamides has been reported with KDR inhibitory activity. In order to demonstrate the pharmacokinetics and the relationship between the structures and their inhibition of KDR, 3DQSAR and in silico ADME/T analysis were performed on a dataset of 13 compounds. Quantum chemical parameters such as LUMO energy, HOMO energy, ionization energy (I), electron affinity (A), chemical potential $(\mu)$, hardness $(\eta)$ and electrophilicity $(\chi)$ of the compounds are calculated by using semi-empirical SCF-MO method at PM3 level of theory and various SlogP descriptors from MOE software. In silico ADME/T analysis was performed by using different software's Discovery Studio 2.5, TOPKAT and QikProp 4.3. From in silico ADME and Toxicity studies, it was revealed that selected derivatives have good oral absorption rate and metabolism with no BBB penetration. QSTR (Quantitative Structure Toxicity Relationship) studies by using TOPAK in various computational animal models, showed high $\mathrm{LD}_{50}$ values and the compounds are found to be noncarcenogenic. Moreover, three different QSAR models were generated by Partial Least Squares (PLS) Regression method having correlation coefficient $\left(\mathrm{Q}^{2}\right)$ of $0.86382,0.84372$, and 0.82629 , respectively. These models conclude a significant relationship of KDR inhibition with dipole moment (D), LUMO energy (ELUMo), hardness $(\eta)$ and electrophilicity $(\chi)$, and hydrophobicity of compounds, regarding N-4chlorophenylnaphthamides.
\end{abstract}

\section{INTRODUCTION}

New blood vessels formation from existing vasculature is termed as angiogenesis, a crucial pathway for tissue repair, growth and embryogenesis (Cherrington et al., 2000, Ferrara, 2002, Harmange et al., 2008) and associated with different types of pathological processes which include metastasis (Liotta et al., 1991), tumor growth (Folkman, 1972), psoriasis (Detmar, 2000), ocular neovascularization (Aiello et al., 1994), rheumatoid

\footnotetext{
* Corresponding Author

S. M. Zahid Hosen, Molecular Modeling \& Drug Design Laboratory (MMDDL), Pharmacology Research Division, Bangladesh Council of Scientific \& Industrial Research (BCSIR), Chittagong-4220, Bangladesh. Email: smzahidhosen @ bcsir.gov.bd
}

arthritis (Walsh and Haywood, 2001), inflammation (Fava, 1994). Vascular endothelial growth factor (VEGFa) and its receptor tyrosine kinases VEGFR-2 or kinase insert domain receptor (KDR) and VEGFR-1 were reported as key regulators for angiogenesis (Terman et al., 1992). Briefly, downstream pathways are initiated by the accumulation of receptor dimerization and intercellular autophosphorylation, when VEGF binds with its receptor VEGFRs. As a corollary, vascular permeability, endothelial cell proliferation, survival and migration are increased and resulted in tumor growth and metastasis (Kilari et al., 2013; Quentmeier et al., 2012). Moreover, excessive expression of VEGFR is related to the poor prognosis and aggressiveness of the tumor in most of the cancers and therefore, inhibition of KDR-VEGF binding activity is 
one of the major strategies in cancer drug development (Li et al., 2016). Some successful strategies to inhibit the angiogenesis have been effectively expressed in preclinical and clinical layout. These approaches include VEGF soluble decoy receptors (Cardones and Banez, 2006), antibodies directed against VEGF (D'Adamo et al., 2005), and small molecules that inhibit KDR (Adjei, 2007; Beebe et al., 2003; Gingrich et al., 2003; Harmange et al., 2008; Hennequin et al., 2002; Ruggeri et al., 2003; Ryan and Wedge, 2005; Sun et al., 2003; Thomas et al., 2003). From these observations and ever increasing demands of these biological active KDR inhibitors, we subjected some novel N-4chlorophenylnaphthamide based KDR inhibitors to study their QSAR and QSTR profiles.

The approach are used based on the popular quantum based drug design methodologies to describe the ADME/T profiles and activity by taking some quantum chemical parameters including dipole moment (D), LUMO energy ( $\mathrm{E}_{\mathrm{LUMO}}$ ), hardness $(\eta)$ and electrophilicity $(\chi)$, along with hydrophobic descriptor SLogP.

\section{MATERIALS AND METHODS}

\section{Dataset preparation}

Thirteen N-4-chlorophenylnaphthamides with KDR inhibitory activity were used in systemic ADME/T and QSAR analysis are represented in Table 1. The molecules were drawn in Symyx Drawer and then subjected to generate 3D molecular structure by energy minimizing them with MMFF force field (Halgren, 1996) followed by considering distance-dependent dielectric constant of 1.0 and convergence criterion of 0.01 $\mathrm{kcal} / \mathrm{mol} \mathrm{A}$.

\section{Prediction of ADME descriptors and toxicity}

The major reason for the failure of most of the drug candidates during clinical trial are poor ADME and high toxicity profile. Thus an important aspect of drug discovery is to evaluate critical physio-chemical as well as toxicity profile in initial stages of drug discovery (Ray et al., 2010). Hence, to select a potential drug candidate, all 13 hypothetical ligands were screened on the basis of ADME and toxicity filter using QikProp 4.3 from Schrodinger 2013 software. QiKProp used Maestro-formatted .mae files (also known as 3D SD files) as input. Output of QikProp showed a number of principal descriptors and ADME properties as shown in Table S1 (Choudhary et al., 2015). Lipinski rule of 5 was applied on the ligands and hits which were having more than 1 violation were rejected. Various other physicochemical properties were also calculated, represented by different descriptors such as molar weight (MW), number of rotatable bonds (NRB), lipophilicity parameter $[\log \mathrm{P}(\mathrm{o} / \mathrm{w})]$, number of hydrogen bond acceptors (HBA), number of hydrogen bond donors (HBD), total polar sur-face area (TPSA), solubility ( $\log s$ ), solvent-accessible surface area (SASA), skin permeability (log $\mathrm{Kp}$ ), binding to human serum albumin (log Khsa), blood-brain partition coefficient $(\log \mathrm{BB})$, apparent MDCK cell permeability (affyPMDCK), apparent Caco-2 cell permeability (affyPCaco), percentage human oral absorption (Dash et al., 2015). Ideal ranges of various descriptors calculated with the reference to $95 \%$ of drugs are presented in Table S1.

Table 1: Descriptions and activity of the selected KDR inhibitors including name, structure and predicted activity by using three different models.

\begin{tabular}{|c|c|c|c|c|c|c|c|}
\hline \multirow[b]{2}{*}{$\begin{array}{l}\text { Compound } \\
\text { Name }\end{array}$} & \multirow[b]{2}{*}{ Structure } & \multirow[b]{2}{*}{ Compound's Name } & \multirow[b]{2}{*}{$\mathrm{IC}_{50}$} & \multicolumn{4}{|c|}{$\mathrm{pIC}_{50}$} \\
\hline & & & & Observed & $\begin{array}{c}\text { Cal } \\
(D, \text { SLOGP, } \chi)\end{array}$ & $\begin{array}{c}\text { Cal } \\
\text { (D, SLOGP, } \\
\text { LUMO) }\end{array}$ & $\begin{array}{c}\text { Cal } \\
(D, \text { SLOGP, } \\
\eta)\end{array}$ \\
\hline 1 & & $\begin{array}{c}\text { 4-(5-((4- } \\
\text { Chlorophenyl)carbamo } \\
\text { yl)naphthalen-2- } \\
\text { yloxy)-N- } \\
\text { methylpicolinamide }\end{array}$ & 3 & 8.52288 & 8.27259 & 8.26322 & 8.13549 \\
\hline 2 & & $\begin{array}{l}\text { N-(4-Chlorophenyl)-6- } \\
\text { (pyridin-4-yloxy)-1- } \\
\text { naphthamide }\end{array}$ & 5 & 7.74473 & 7.82488 & 7.92529 & 7.83936 \\
\hline 3 & & $\begin{array}{c}\mathrm{N}-(4-C h l o r o p h e n y l)-6- \\
\text { (2- } \\
\text { (methylamino)pyridin- } \\
\text { 4-yloxy)-1- } \\
\text { naphthamide } \\
\end{array}$ & 7 & 8.1549 & 7.72738 & 7.71313 & 7.89485 \\
\hline 4 & & $\begin{array}{l}\mathrm{N}-(4-C h l o r o p h e n y l)-6- \\
\text { (pyrimidin-4-yloxy)-1- } \\
\text { naphthamide }\end{array}$ & 310 & 6.50864 & 6.69023 & 6.59928 & 6.8149 \\
\hline 5 & & $\begin{array}{c}\mathrm{N}-(4-C h l o r o p h e n y l)-6- \\
(2- \\
\text { (methylamino)pyrimid } \\
\text { in-4-yloxy)-1- } \\
\text { naphthamide }\end{array}$ & 46 & 7.33724 & 7.81124 & 7.80144 & 7.73016 \\
\hline
\end{tabular}




\begin{tabular}{|c|c|c|c|c|c|c|}
\hline 6 & $\begin{array}{c}\text { N-(4-Chlorophenyl)-6- } \\
\text { (6- } \\
\text { (methylamino)pyrimid } \\
\text { in-4-yloxy)-1- } \\
\text { naphthamide }\end{array}$ & 20 & 7.69897 & 7.63951 & 7.66307 & 7.54596 \\
\hline 7 & $\begin{array}{l}\text { 6-(7H-Pyrrolo[2,3- } \\
\text { d]pyrimidin-4-yloxy)- } \\
\text { N-(4-chlorophenyl)- } \\
\text { 1-naphthamide }\end{array}$ & 28 & 7.55284 & 7.54616 & 7.54635 & 7.54794 \\
\hline 8 & $\begin{array}{c}\text { N-(4-Chlorophenyl)-6- } \\
\text { (quinolin-4-yloxy)-1- } \\
\text { naphthamide }\end{array}$ & 4 & 8.39794 & 8.29596 & 8.46617 & 8.44174 \\
\hline 9 & $\begin{array}{l}\text { N-(4-Chlorophenyl)-6- } \\
\text { (7-methoxyquinolin-4- } \\
\text { yloxy)-1-naphthamide }\end{array}$ & 1 & 9 & 8.9622 & 8.61254 & 8.53581 \\
\hline 10 & $\begin{array}{l}\text { N-(4-Chlorophenyl)-6- } \\
\quad(6,7- \\
\text { dimethoxyquinolin-4- } \\
\text { yloxy)-1-naphthamide }\end{array}$ & 0.5 & 7.38722 & 7.85128 & 7.9445 & 7.94698 \\
\hline 11 & $\begin{array}{l}\text { N-(4-Chlorophenyl)-6- } \\
\text { (3-fluoro-6,7- } \\
\text { dimethoxyquinolin-4- } \\
\text { yloxy)- } \\
\text { 1-naphthamide }\end{array}$ & 20 & 7.69897 & 7.71085 & 7.7105 & 7.70762 \\
\hline 12 & $\begin{array}{c}\text { 4-(5-((4- } \\
\text { Chlorophenyl)carbamo } \\
\text { yl)naphthalen-2- } \\
\text { yloxy)-7- } \\
\text { methoxyquinoline-6- } \\
\text { carboxamide }\end{array}$ & 0.2 & 9.69897 & 9.94529 & 9.95451 & 10.0802 \\
\hline 13 & $\begin{array}{c}\text { N-(4-Chlorophenyl)-6- } \\
(6,7- \\
\text { dimethoxyquinazolin- } \\
\text { 4-yloxy)-1- } \\
\text { naphthamide }\end{array}$ & 2 & 8.69897 & 8.12471 & 8.20227 & 8.18124 \\
\hline
\end{tabular}

Table S1: The Descriptors used in ADME-T prediction.

\begin{tabular}{|c|c|c|}
\hline Predicted physiochemical properties & Used Descriptors & Ideal Range in $95 \%$ of drugs \\
\hline Predicted molecular weight & MW & $311-650$ \\
\hline Predicted dipole moment & $\mathrm{DM}$ & $0.000-1000.0$ \\
\hline Predicted total molecular solvent accessible surface area & SASA & $300.0-1000.0$ \\
\hline Predicted hydrophobic SASA & FOSA & $0-750$ \\
\hline Predicted hydrophilic SASA & FISA & $7-330$ \\
\hline Predicted carbon Pi SASA & PISA & $0-450$ \\
\hline Predicted weakly polar SASA & WPSA & $0-175$ \\
\hline Predicted number of hydrogen bond donor & HB-donor & $0-6$ \\
\hline Predicted number hydrogen bond acceptor & HB-accept & $2-20$ \\
\hline Predicted octanol/gas partition coefficient & QP $\log$ P OCT & $8.0-35.0$ \\
\hline Predicted water/gas partition coefficient & QP $\log$ P W & $4.0-45.0$ \\
\hline Predicted octanol/water partition coefficient & $\mathrm{QP} \log \mathrm{PO} / \mathrm{W}$ & $-2-6.5$ \\
\hline Predicted aqueous solubility & QP Log S & $-6.5-0.5$ \\
\hline Predicted blood-brain partition coefficient & QPLogBB (Cbrain/Cblood) & $-3.0-1.2$ \\
\hline Predicted skin permeability & QP Log KP & -8.0 to -1.0 \\
\hline Predicted van der walls surface area of polar nitrogen and oxygen atoms & PSA & $7-200$ \\
\hline Predicted human oral absorption & $\mathrm{HOA}$ & $>80 \%$ is high $<25 \%$ is low \\
\hline Predicted number of rotable bond & \#rotor & $<10$ \\
\hline Predicted ionization potential & $\mathrm{IP}(\mathrm{ev})$ & $0.000-9.000$ \\
\hline Predicted apparent MDCK cell permeability in $\mathrm{nm} / \mathrm{sec}$ & AffyPMDCK & $<25$ poor, $>500$ great \\
\hline Predicted apparent Caco-2 cell permeability in $\mathrm{nm} / \mathrm{sec}$ & AffyPCaco & $<25$ poor, $>500$ great \\
\hline Prediction of binding to human serum albumin. & QPLogKhsa & $-1.5-1.5$ \\
\hline Predicted $\mathrm{IC}_{50}$ in vitro & Log HERG & Concern below -5 \\
\hline Lipinski's rule of five & Rule of 5 & $\operatorname{Max} 4$ \\
\hline
\end{tabular}




\section{ADME/T prediction in Discovery Studio}

For additional validation purpose, Discovery studio 2.5 (Accelrys, San Diego, CA, USA) was used to describe absorption, distribution, metabolism, elimination, and toxicity (ADME/T) properties by using ADME/T descriptors module. Based on the existing information of drug, six mathematical models are used in the modules to predict quantitatively the properties by a using set of rules/keys (Table S2) that specify threshold ADME/T characteristics for the chemical structure of the molecules. TOPKAT predictor was also selected, where the models that were used to calculate using TOPKAT are Rat Oral $\mathrm{LD}_{50}$ (v3.1), Daphnia $\mathrm{EC}_{50}(\mathrm{v} 3.1)$.

Table S2: ADMET descriptors and their rules/keys.

\begin{tabular}{|c|c|c|}
\hline \multicolumn{3}{|c|}{ ADMET absorption level (human intestinal absorption) } \\
\hline Level & & Description \\
\hline 0 & & Good absorption \\
\hline 1 & & Moderate absorption \\
\hline 2 & & Low absorption \\
\hline 3 & & Very low absorption \\
\hline \multicolumn{3}{|c|}{ ADMET aqueous solubility level } \\
\hline Level & Value & Description \\
\hline \multirow[t]{2}{*}{0} & $\log$ (molar solubility) & Extremely low \\
\hline & $<-8.0$ & \\
\hline \multirow[t]{2}{*}{1} & $-8.0<\log (\mathrm{molar}$ & No, very low, but possible \\
\hline & solubility) $<-6.0$ & \\
\hline \multirow[t]{2}{*}{2} & $-6.0<\log ($ molar & Yes, low \\
\hline & solubility) $<-4.0$ & \\
\hline \multirow[t]{2}{*}{3} & $-4.0<\log ($ molar & Yes, good \\
\hline & solubility) $<-2.0$ & \\
\hline \multirow[t]{2}{*}{4} & $-2.0<\log ($ molar & Yes, optimal \\
\hline & solubility) $<0.0$ & \\
\hline 5 & $0.0<\log$ (molar solubility) & No, too soluble \\
\hline \multirow[t]{3}{*}{6} & -1000 & Warning: molecules with \\
\hline & & one or more unknown \\
\hline & & AlogP98 types \\
\hline \multicolumn{3}{|c|}{ ADMET (blood brain barrier penetration level) BBB } \\
\hline \multicolumn{2}{|c|}{ Level } & Description \\
\hline \multicolumn{2}{|l|}{0} & Very High \\
\hline \multicolumn{2}{|l|}{1} & High \\
\hline \multicolumn{2}{|l|}{2} & Medium \\
\hline \multicolumn{2}{|l|}{3} & Low \\
\hline \multirow[t]{2}{*}{4} & & Undefined \\
\hline & & Warning: molecules with \\
\hline \multirow[t]{2}{*}{5} & & one or more unknown \\
\hline & & AlogP calculation \\
\hline \multirow{2}{*}{\multicolumn{2}{|c|}{ Predictedclass }} & D6 \\
\hline & & Value \\
\hline \multicolumn{2}{|l|}{0} & Noninhibitor \\
\hline \multicolumn{2}{|l|}{1} & Inhibitor \\
\hline \multirow{2}{*}{\multicolumn{2}{|c|}{ Predictedclass }} & xicity \\
\hline & & Value \\
\hline \multicolumn{2}{|l|}{0} & Nontoxic \\
\hline \multicolumn{2}{|l|}{1} & Toxic \\
\hline \multicolumn{3}{|c|}{ ADMET (plasma protein binding level) PPB } \\
\hline Level & & Description \\
\hline 0 & & Binding is $<90 \%$ \\
\hline 1 & & Binding is $\geq 90 \%$ \\
\hline 2 & & Binding is $\geq 95 \%$ \\
\hline
\end{tabular}

\section{Quantum Descriptors calculation and QSAR Analysis}

All compounds were subjected to geometry optimization by using semi-empirical SCF-MO method, implemented in MOPAC 7.0. Here, basis set PM3 was used to calculate the quantam descriptors. The following quantum chemical descriptors were considered: the energy of the highest occupied molecular orbital $\left(\mathrm{E}_{\mathrm{HOMO}}\right)$, the lowest unoccupied molecular orbital $\left(\mathrm{E}_{\mathrm{LUMO}}\right)$, the band gap energy $(\Delta \mathrm{E})$, the dipole moment (D), Electrophilicity $(\chi)$, Global Hardness $(\eta)$. Using Koopmans' approximation, and ionization energy (I) and electron affinity (A) can be expressed in terms of the energies of the highest occupied $\left(\mathrm{E}_{\mathrm{HOMO}}\right)$ and the lowest unoccupied molecular orbital $\left(\mathrm{E}_{\mathrm{LUMO}}\right)$ as:

$$
\begin{gathered}
\text { Ionization potential, } \mathrm{I}=-\mathrm{E}_{\text {HOмо }} \\
\text { Electron affinity, } \mathrm{A}=-\mathrm{E}_{\mathrm{LUMO}}
\end{gathered}
$$

Electrophilicity index is defined $(\chi)$ as a measure of the decrease in energy due to the maximal transfer of electrons from a donor to an acceptor system and is given as:

$$
\chi=\mu / 2 \eta
$$

Where, $\mu$ and $\eta$ are the chemical potential and hardness, respectively. Chemical potential and hardness can be expressed in terms of ionization energy (I) and electron affinity (A) as given below:

$$
\begin{aligned}
& \eta=[\text { HOMO } \varepsilon-\text { LUMO } \varepsilon] / 2 \\
& \mu=\left(\frac{\partial E}{\partial N}\right)_{\nu(r)} \approx-\frac{I+A}{2}
\end{aligned}
$$

After that, 2D molecular descriptors, SlogPs (according to various subdivided accessible van der Waals surface area) were calculated by the program MOE (MOE software: Chemical computing group's molecular operating environment (MOE) software, version 2014.0901). After that, PLS QSAR (Geladi and Kowalski, 1986, Helland, 1988) analysis was performed to determine the relationship between these molecular descriptors and biological activity of the compounds. The maximum condition number of the principal component transform of the correlation matrix $S$, the condition limit, was set at $1.0 \times 10^{6}$ which is a very high setting. The leave-one-out cross validation (LOO-CV) scheme was used to validate the models and the correlation coefficient $\left(\mathrm{Q}^{2}\right)$ and root-mean-square error (RMSE) were reported.

\section{RESULTS AND DISCUSSIONS}

\section{ADME and toxicity screening}

All 13 hypothetical compounds were screened for "druglikeliness and "non-toxic" profile on the basis of various parameters of the software QikProp. ADME filter lead to 13 drugs like hits. The ADME prediction studies data of 13 hits are shown in Table 2. The range of various important parameters were predicted, like molecular weight lied in range between 374.826 and 502.928, the value of total solvent accessible surface area (SASA) ranged 645.681-802.389, estimated number of hydrogen 
bonds donated (Donor $\mathrm{HB}$ ) by the solute to water molecules in an aqueous solutions was found between 1 and 3, estimated number of hydrogen bonds accepted (accept HB) by the solute to water molecules in an aqueous solutions ranged $4-7.25$, predicted octanol/gas par-tition coefficient (QPlogPoct) ranged 18.43927.16, predicted water/gas partition coefficient ( $\mathrm{QPlog} \mathrm{Pw})$ ranged 10.268-16.232, predicted octanol/water partition coefficient $(\mathrm{QPlogPo} / \mathrm{w})$ ranged 3.602-6.513, predicted aqueous solubility, $\log \mathrm{S}(\mathrm{QP} \log \mathrm{S})$ ranged -5.684 to -8.177 , predicted brain/blood partition coefficient (QPlogBB) ranged -0.144 to -1.429 , and Lipinski violations were $\leq 2$. Results of ADME-T studies reveal that compounds from $\mathbf{1}$ to $\mathbf{1 3}$ can be considered best, having druglikliness as well as non-toxicity profile.

\section{ADME/T Prediction by TOPAK}

The use of In silico approaches to predict ADME/T properties is considered as a first step in the direction to analyze the new chemical entities to reduce wasting time on lead compounds which would be toxic or metabolized by the body into an inactive form and unable to pass through membranes, and some results for this analysis are herein depicted in Table 3 along with ADME/T descriptors and their rules/keys are tabulated in Table S2.The ADME/T profile of all the molecules under investigation was forecasted by means of six pre calculated ADME/T models provided by the Discovery Studio 2.5 program. Lipophilicity could be estimated as the $\log$ of the partition coefficient between noctanol and water $(\log \mathrm{P})$. Though $\log \mathrm{P}$ is generally used to determine a compound's lipophilicity, the fact that $\log \mathrm{P}$ is a ratio creates a concern about the use of $\log \mathrm{P}$ to score hydrophilicity and hydrophobicity (Hughes et al., 2008). Thus the information of $\mathrm{H}-$ bonding prominences as obtained by calculating PSA could be taken into consideration along with $\log \mathrm{P}$ calculation (Egan et al., 2000). Therefore, a model with descriptors AlogP98 and PSA 2D were taken into consideration for the accurate prediction for the cell permeability of compounds. According to the model for a compound to have an optimum cell permeability should follow the evaluation criteria (PSA $<140 \mathrm{~A}^{\circ 2}$ and $\left.\mathrm{A} \log \mathrm{P} 98<5\right)$ (Egan et al., 2000).

Table 2: ADME-T descriptors of the compound calculated from QikProp.

\begin{tabular}{|c|c|c|c|c|c|c|c|c|c|c|c|c|c|c|c|c|}
\hline 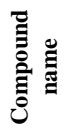 & $\sum$ & 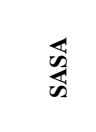 & 全 & 苂 & $\begin{array}{l}\frac{3}{0} \\
\frac{0}{0} \\
\frac{0}{2} \\
\frac{0}{2}\end{array}$ & $\begin{array}{l}20 \\
\frac{0}{0} \\
\frac{0}{2}\end{array}$ & $\begin{array}{l}\frac{a}{00} \\
\frac{0}{\partial} \\
\bar{\sigma}\end{array}$ & $\begin{array}{l}\frac{F}{2} \\
\frac{a}{0} \\
\frac{0}{0} \\
\overline{0}\end{array}$ & 总 & 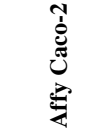 & $\begin{array}{l}00 \\
000 \\
\frac{0}{\partial}\end{array}$ & 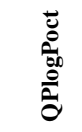 & $\begin{array}{l}3 \\
\frac{0}{0} \\
0 \\
0\end{array}$ & $\frac{\pi}{0}$ & ש్ & $\begin{array}{l}n \\
\stackrel{0}{0}\end{array}$ \\
\hline 1 & 417.851 & 700.599 & 3 & 6.5 & 3.602 & -5.99 & -2.617 & 0.391 & 246.755 & 227.703 & -1.389 & 23.459 & 15.402 & 90.23 & 105.509 & 0 \\
\hline 2 & 374.826 & 651.13 & 1 & 4.5 & 4.956 & -6.15 & -0.588 & 0.672 & 2923.974 & 2242.509 & -0.191 & 18.439 & 10.268 & 100 & 53.16 & 0 \\
\hline 3 & 403.867 & 706.46 & 2 & 4.5 & 5.297 & -6.844 & -0.772 & 0.823 & 2502.012 & 1941.401 & -0.359 & 21.084 & 11.383 & 100 & 64.318 & 1 \\
\hline 4 & 375.813 & 645.681 & 1 & 5.5 & 4.22 & -5.684 & -1.219 & 0.435 & 1514.856 & 1220.398 & -0.473 & 18.622 & 11.202 & 100 & 66.347 & 0 \\
\hline 5 & 404.855 & 702.79 & 2 & 5.5 & 4.624 & -6.457 & -1.313 & 0.61 & 1424.41 & 1152.841 & -0.617 & 21.138 & 12.327 & 100 & 76.818 & 0 \\
\hline 6 & 404.855 & 692.903 & 2 & 5.5 & 4.443 & -6.285 & -1.702 & 0.597 & 940.088 & 784.881 & -0.779 & 20.866 & 12.298 & 100 & 79.286 & 0 \\
\hline 7 & 414.85 & 676.781 & 2 & 5 & 4.544 & -6.327 & -1.725 & 0.684 & 781.341 & 661.434 & -0.768 & 20.965 & 12.461 & 100 & 80.817 & 0 \\
\hline 8 & 424.885 & 715.766 & 1 & 4 & 6.121 & -7.505 & -0.195 & 1.124 & 3490.211 & 2641.573 & -0.144 & 20.166 & 10.37 & 100 & 52.128 & 1 \\
\hline 9 & 454.912 & 752.592 & 1 & 4.75 & 6.212 & -7.719 & -0.293 & 1.129 & 3503.646 & 2650.982 & -0.218 & 21.12 & 10.597 & 100 & 60.396 & 1 \\
\hline 10 & 484.938 & 797.04 & 1 & 5.5 & 6.396 & -8.065 & -0.37 & 1.171 & 3370.03 & 2557.287 & -0.316 & 22.375 & 10.957 & 100 & 64.821 & 1 \\
\hline 11 & 502.928 & 794.337 & 1 & 5.5 & 6.513 & -8.177 & -0.509 & 1.181 & 5373.213 & 2559.34 & -0.219 & 22.273 & 10.694 & 100 & 65.178 & 2 \\
\hline 12 & 497.937 & 802.389 & 3 & 7.25 & 4.694 & -7.321 & -2.217 & 0.737 & 329.67 & 297.696 & -1.429 & 27.16 & 16.232 & 100 & 110.338 & 0 \\
\hline 13 & 485.926 & 789.7 & 1 & 6.5 & 5.682 & -7.535 & -0.828 & 0.908 & 2117.295 & 1663.539 & -0.535 & 22.446 & 11.875 & 100 & 78.508 & 1 \\
\hline
\end{tabular}

Data indicate the descriptor calculated from QIKProp 4.3. where each descriptor represent one physicochemical properties for "druglikeliness" such as MW molecular weight; SASA- Solvent Assessable Surface Area; HBD- no. of H bond donor; HBA- no. of H bond acceptor; QPlogPo/w- predicted octanol/water partition coefficient; QPlogS- predicted aqueous solubility; QPlogKp- predicted skin permeability; QPlogKhsa- predicted human serum albumin binding; Affy MDCK- Apparent MDCK cell permeability; AffyCaco- Apparent Caco-2 cell permeability; QPlogBB- predicted brain/blood partition coefficient; QPlogPoctpredicted octanol/gas partition coefficient; QPlogPw-predicted water/gas partition coefficient.\%HOA- Percentage Human Oral Absorption; PSA- Total Polar Surface Area; Rule of 5-Lipinski violations.

Table 3: Computer aided ADME/T screening Of 13 Compounds

\begin{tabular}{|c|c|c|c|c|c|c|c|c|c|c|c|c|c|}
\hline 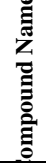 & 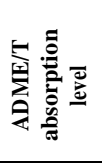 & 震离 & 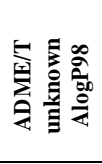 & 空令 & 窐离 & $\sum^{5}$ & 震总 & 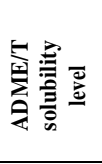 & 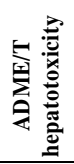 & 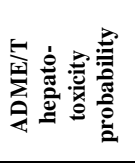 & 零令 & 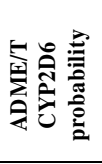 & 氛离 \\
\hline 1 & 0 & 4.027 & 0 & 94.142 & 4 & - & -5.842 & 2 & 1 & 0.986 & 0 & 0.465 & 1 \\
\hline 2 & 0 & 4.597 & 0 & 50.302 & 4 & - & -5.966 & 2 & 1 & 0.98 & 1 & 0.712 & 2 \\
\hline 3 & 0 & 4.944 & 0 & 63.112 & 1 & 0.376 & -6.28 & 1 & 1 & 0.986 & 1 & 0.673 & 1 \\
\hline 4 & 0 & 4.508 & 0 & 61.563 & 1 & 0.265 & -5.967 & 2 & 1 & 0.98 & 1 & 0.722 & 2 \\
\hline 5 & 0 & 4.837 & 0 & 74.373 & 1 & 0.164 & -6.349 & 1 & 1 & 0.98 & 1 & 0.712 & 2 \\
\hline 6 & 0 & 4.855 & 0 & 74.373 & 1 & 0.17 & -6.365 & 1 & 1 & 0.98 & 1 & 0.712 & 2 \\
\hline 7 & 0 & 4.861 & 0 & 76.618 & 1 & 0.136 & -6.834 & 1 & 1 & 0.986 & 1 & 0.594 & 1 \\
\hline 8 & 1 & 5.934 & 0 & 50.302 & 0 & 0.884 & -7.494 & 1 & 1 & 0.986 & 1 & 0.574 & 2 \\
\hline 9 & 1 & 5.917 & 0 & 59.232 & 0 & 0.738 & -7.375 & 1 & 1 & 0.986 & 1 & 0.564 & 2 \\
\hline 10 & 1 & 5.901 & 0 & 68.162 & 4 & - & -7.268 & 1 & 1 & 0.973 & 1 & 0.603 & 2 \\
\hline 11 & 1 & 6.106 & 0 & 68.162 & 4 & - & -7.444 & 1 & 1 & 0.98 & 1 & 0.653 & 2 \\
\hline 12 & 1 & 4.919 & 0 & 103.072 & 4 & - & -6.915 & 1 & 1 & 0.98 & 1 & 0.603 & 1 \\
\hline 13 & 1 & 5.812 & 0 & 79.423 & 4 & - & -7.247 & 1 & 1 & 0.973 & 1 & 0.554 & 2 \\
\hline
\end{tabular}


Table 4: Rat oral $\mathrm{LD}_{50}$ - Rat oral lethal dose 50

\begin{tabular}{|c|c|c|}
\hline \multirow[t]{2}{*}{ Compound Name } & \multicolumn{2}{|c|}{ Rat Oral LD s0 $_{1 e}$ Hetearomatic Model } \\
\hline & Computed Rat Oral LD $_{50}$ & 95\% Confidence Limits \\
\hline 1 & $476.9 \mathrm{mg} / \mathrm{kg}$ & $75.1 \mathrm{mg} / \mathrm{kg} \& 3 \mathrm{~g} / \mathrm{kg}$ \\
\hline 2 & $478.7 \mathrm{mg} / \mathrm{kg}$ & $79.6 \mathrm{mg} / \mathrm{kg} \& 2.8 \mathrm{~g} / \mathrm{kg}$ \\
\hline 3 & $504.1 \mathrm{mg} / \mathrm{kg}$ & $85 \mathrm{mg} / \mathrm{kg} \& 3 \mathrm{~g} / \mathrm{kg}$ \\
\hline 4 & $474.5 \mathrm{mg} / \mathrm{kg}$ & $81.6 \mathrm{mg} / \mathrm{kg} \& 2.8 \mathrm{~g} / \mathrm{kg}$ \\
\hline 5 & $595.2 \mathrm{mg} / \mathrm{kg}$ & $101.5 \mathrm{mg} / \mathrm{kg} \& 3.5 \mathrm{~g} / \mathrm{kg}$ \\
\hline 6 & $576.9 \mathrm{mg} / \mathrm{kg}$ & $98 \mathrm{mg} / \mathrm{kg} \& 3.4 \mathrm{~g} / \mathrm{kg}$ \\
\hline 7 & 404 mg/kg & $68.9 \mathrm{mg} / \mathrm{kg} \& 2.4 \mathrm{~g} / \mathrm{kg}$ \\
\hline 8 & $341.1 \mathrm{mg} / \mathrm{kg}$ & $57.5 \mathrm{mg} / \mathrm{kg} \& 2 \mathrm{~g} / \mathrm{kg}$ \\
\hline 9 & $297 \mathrm{mg} / \mathrm{kg}$ & $50 \mathrm{mg} / \mathrm{kg} \& 1.8 \mathrm{~g} / \mathrm{kg}$ \\
\hline 10 & $139 \mathrm{mg} / \mathrm{kg}$ & $22.8 \mathrm{mg} / \mathrm{kg} \& 848 \mathrm{mg} / \mathrm{kg}$ \\
\hline 11 & $312.5 \mathrm{mg} / \mathrm{kg}$ & $51.6 \mathrm{mg} / \mathrm{kg} \& 1.9 \mathrm{~g} / \mathrm{kg}$ \\
\hline 12 & $229.2 \mathrm{mg} / \mathrm{kg}$ & $35 \mathrm{mg} / \mathrm{kg} \& 1.5 \mathrm{~g} / \mathrm{kg}$ \\
\hline 13 & $161.4 \mathrm{mg} / \mathrm{kg}$ & $26.6 \mathrm{mg} / \mathrm{kg} \& 980.8 \mathrm{mg} / \mathrm{kg}$ \\
\hline
\end{tabular}

Table 5: Daphnia $\mathrm{EC}_{50}$ - Daphnia effective concentration 50

\begin{tabular}{|c|c|c|}
\hline \multirow[t]{2}{*}{ Compound Name } & \multicolumn{2}{|c|}{ Model: Daphnia $\mathrm{EC}_{50}(\mathbf{v 3 . 1})$} \\
\hline & Computed $\mathrm{EC}_{50}$ values & 95\% Confidence Limits \\
\hline 1 & $5.4 \mathrm{ug} / \mathrm{l}$ & 731.1ng/l\&39.2ug/l \\
\hline 2 & $10.6 \mathrm{ug} / \mathrm{l}$ & $1.8 \mathrm{ug} / \mathrm{l} \& 1.9 \mathrm{mg} / \mathrm{l}$ \\
\hline 3 & $347.5 \mathrm{ug} / 1$ & $58.1 \mathrm{ug} / 1 \& 2.1 \mathrm{mg} / 1$ \\
\hline 4 & $13.6 u g / 1$ & $2.2 \mathrm{ug} / 1 \& 83.2 \mathrm{ug} / 1$ \\
\hline 5 & $299.5 \mathrm{ug} / 1$ & $50 \mathrm{ug} / 1 \& 1.8 \mathrm{mg} / 1$ \\
\hline 6 & $325.7 \mathrm{ug} / 1$ & $54.5 \mathrm{ug} / 1 \& 1.9 \mathrm{mg} / 1$ \\
\hline 7 & $21.9 \mathrm{ug} / 1$ & $3.3 \mathrm{ug} / 1 \& 146.3 \mathrm{ug} / 1$ \\
\hline 8 & $25.4 \mathrm{ug} / 1$ & $3.7 \mathrm{ug} / 1 \& 175.8 \mathrm{ug} / 1$ \\
\hline 9 & $165.1 \mathrm{ug} / 1$ & $24.3 \mathrm{ug} / 1 \& 1.1 \mathrm{mg} / 1$ \\
\hline 10 & 19.6ug/1 & $2.5 \mathrm{ug} / 1 \& 152.2 \mathrm{ug} / 1$ \\
\hline 11 & $1.8 \mathrm{ug} / 1$ & 204.5ng/1\&16.3ug/1 \\
\hline 12 & $43.3 \mathrm{ug} / \mathrm{l}$ & 5.3ug/l\&352.8ug/1 \\
\hline 13 & $18.8 \mathrm{ug} / 1$ & $2.4 \mathrm{ug} / 1 \& 146.1 \mathrm{ug} / 1$ \\
\hline
\end{tabular}

All the compounds showed polar surface area (PSA) $<140 \mathrm{~A}^{\circ 2}$. Considering the AlogP98 criteria, all inhibitors had AlogP98 value $<5$. Table 2 shows that among 13 compounds, 6 compounds have undefined values for BBB penetration levels (4 as mentioned in Table 2) with the exception of 7 compound having very high and high value (level 0 and 1 ) BBB penetration level. The aqueous solubility plays an essential role in the bioavailability of the candidate, all of them having low aqueous solubility level shown as Table S2.

All compounds have been predicted to have hepatotoxicity level of 1 which is toxic to liver and lower rate of first pass metabolism. All compounds exhibit as an inhibitors with respect to CYP2D6 liver (with reference to Table S1) except compound $\mathbf{1}$ shown in Table 3.

This indicates that they are less metabolized in Phase-I metabolism except one. Finally, the ADME/T plasma protein binding property prediction denotes that for compounds $1,2,3,7$, 12 binding is $\geq 90 \%$ and rest of them binding is $\geq 95 \%$ respectively, (refer to Table $\mathrm{S} 1$ ), clearly suggesting that all of them have good bioavailability and are not likely to be highly bound to carrier proteins in the blood. The Rat Oral $\mathrm{LD}_{50}$ values of all the compounds are within the Optimum Prediction Space (OPS). And the $\mathrm{LD}_{50}$ values are satisfactory. These high $\mathrm{LD}_{50}$ values indicate higher safety of these compounds (Table 4). The values obtained from daphnia $\mathrm{EC}_{50}$ model show remarkable $\mathrm{EC}_{50}$ values (Table 5).

\section{QSAR models for inhibitor activity}

The quantum chemical descriptors calculation that obtained from geometry optimization like, dipole moment, HOMO energy, LUMO energy, ionization energy, electron affinity, chemical potential, hardness, and electrophilicity of thirteen molecules are represented in Table S3. Parameters like dipole moment, LUMO energy, hardness and electrophilicity were considered to predict model, since these parameters are widely used to describe the stability, toxicity and reactivity of the chemical compounds (Kapur et al., 2000). We also considered the hydrophobicity, as many authors reported the quantitative correlation of toxicity, activity and metabolism of the compounds with the octanol-water distribution coefficient descriptor (Bundy et al., 2001, Ren and Frymier, 2002, Worgan et al., 2003). In order to establish a better predictability, PLS based QSAR study was performed by considering experimentally observed values $\left(\mathrm{pIC}_{50}\right)$, along with various descriptors, are presented in Tables 1. According to the analysis, the best model included the following predictors: SLogP, dipole moment (D), and electrophilicity $(\chi)$. The model is expressed in the Eq. 1.

$\mathrm{pIC}_{50}=-6.21625+0.18964(\mathrm{D})+0.55132(\chi)+$ $0.05674($ SlogP_VSA9 $)+0.00000($ SlogP_VSA 8$)+$ $0.01887($ SlogP_VSA7 $)+0.00000($ SlogP_VSA6 $)+$ $0.02588($ SlogP_VSA5 $)+0.20657($ SlogP_VSA4 $)+$ 


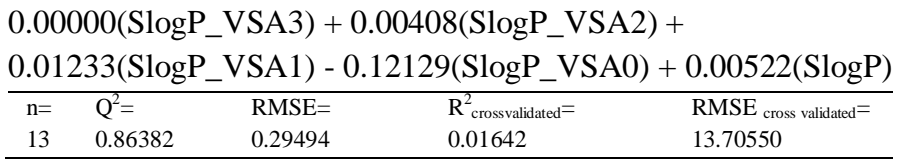

The model considers the higher contributions of SLogP, dipole moment (D) and electrophilicity $(\chi)$ with inhibitory activity. A plot of the predicted activity versus experimental activity for molecules using training set for model of KDR inhibition is shown in Figure 1.

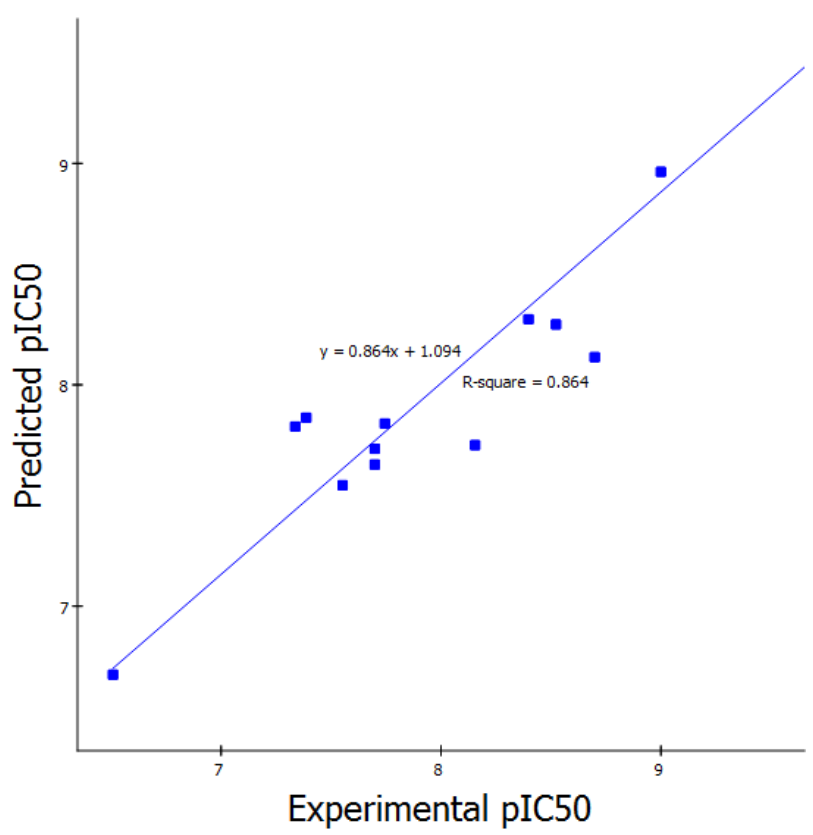

Fig. 1: Calculated and observed activity using SLogP, dipole moment (D), and electrophilicity $(\chi)$ descriptors.

Regarding these descriptors, another best model was observed in case of LUMO energy, in conjunction with Dipole moment (D) and SLogP. The plot of predicted activity values $\left(\mathrm{pIC}_{50}\right)$ versus that predicted on the basis of regression equation for a complete set of inhibitors is presented in Figure 2.

$\mathrm{pIC}_{50}=-7.88749+0.07002\left(\mathrm{~S} \log \mathrm{P}_{-} \mathrm{VSA} 9\right)+$

$0.00000\left(\right.$ S $\left.l o g P \_V S A 8\right)+0.02555\left(\right.$ S $\left.l o g P \_V S A 7\right)+0.00010$

$($ SlogP_VSA6) + 0.21801(SlogP_VSA4 $)+0.03065($ SlogP_VSA5 $)$

$+0.00000($ SlogP_VSA3 $)-0.19162(\mathrm{~S} \log \mathrm{P})-0.13445\left(\mathrm{~S} \log \mathrm{P} \_\mathrm{VSA}\right)$

$+0.01493($ SlogP_VSA1 $)+0.01656($ SlogP_VSA2 $)+0.18532(\mathrm{D})$

- 3.23828(LUMO)

\begin{tabular}{lllll}
\hline $\mathrm{n}=$ & $\mathrm{Q}^{2}=$ & $\mathrm{RMSE}=$ & $\mathrm{R}_{\text {crossvalidated }}^{2}=$ & $\mathrm{RMSE}_{\text {cross validated }}=$ \\
13 & 0.84372 & 0.31595 & 0.01635 & 13.99739 \\
\hline
\end{tabular}

The contribution of global hardness $(\eta)$ was also found in the correlation of biological activity, along with dipole moment (D) and $\mathrm{S} \log \mathrm{P}$, which is shown in eq. (3). The correlations that found in eq. 3, is rendered in Figure 3.
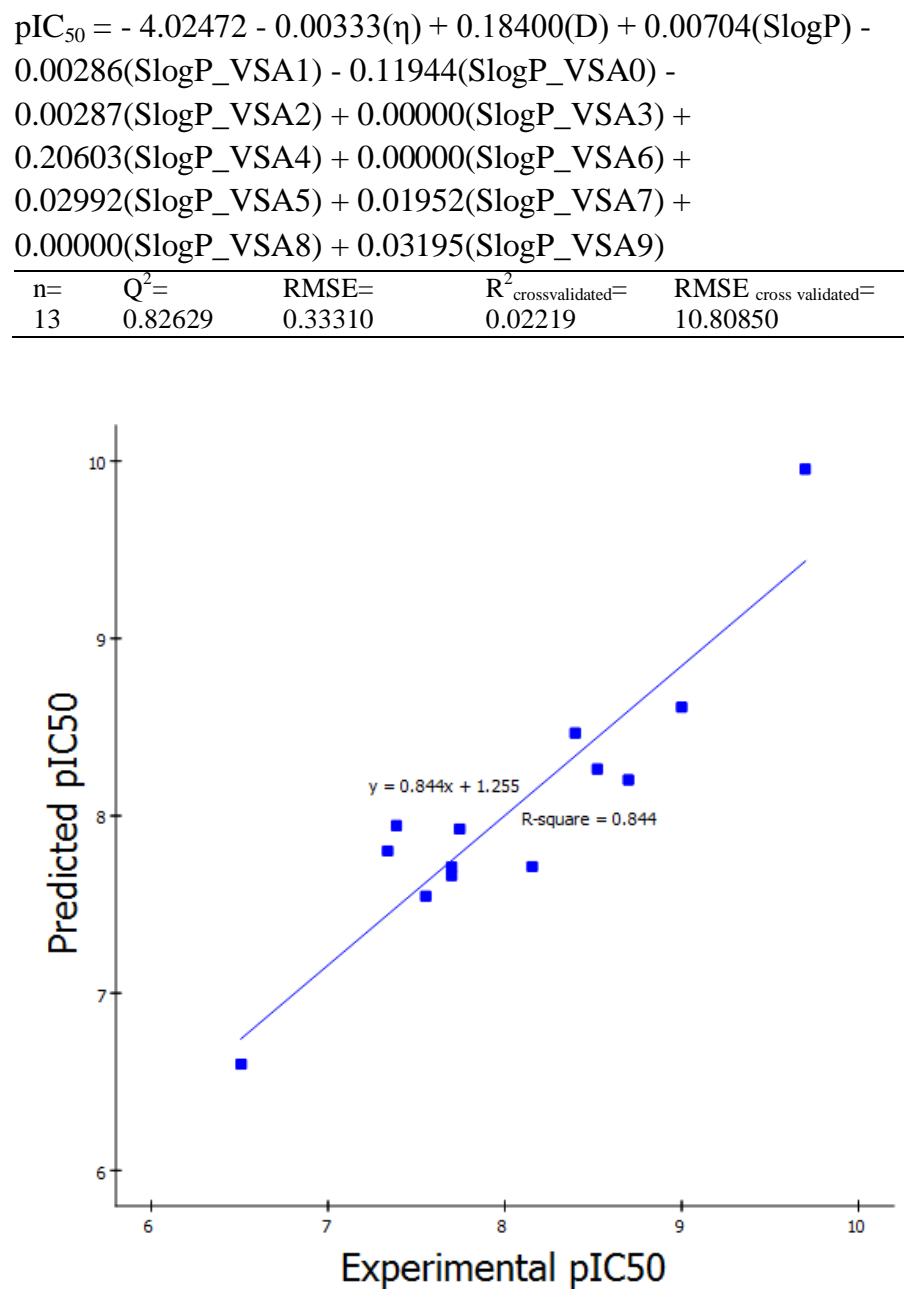

Fig. 2: Observed and calculated $\mathrm{pIC}_{50}$ values using $\mathrm{SLogP}$, dipole moment (D), and $\mathrm{E}_{\mathrm{LUMO}}$ energy descriptors.

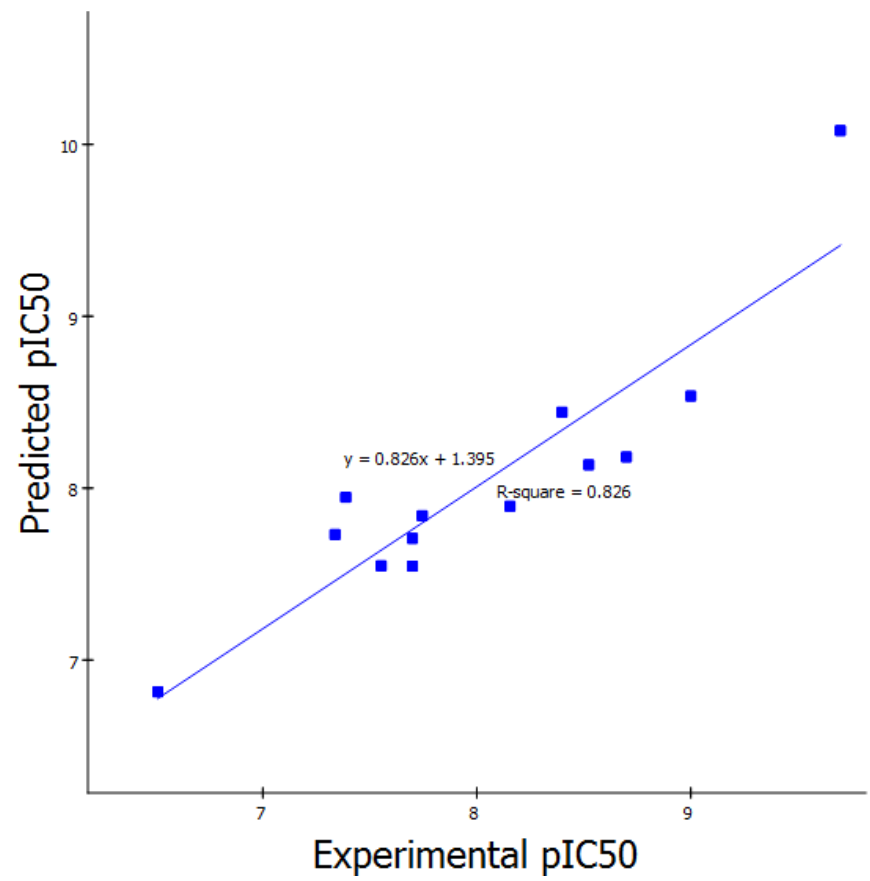

Fig. 3: Observed and calculated $\mathrm{pIC}_{50}$ values using $\mathrm{SLogP}$, dipole moment (D), and hardness $(\eta)$ descriptors. 
Table S3: Calculated quantum descriptors of selected KDR inhibitors.

\begin{tabular}{|c|c|c|c|c|c|c|c|c|c|}
\hline $\begin{array}{l}\text { Compound } \\
\text { Name }\end{array}$ & $\begin{array}{c}\text { Dipole } \\
\text { Moment (D) }\end{array}$ & $\begin{array}{l}\text { НОМО } \\
\left(\mathrm{E}_{\text {Номо }}\right)\end{array}$ & $\begin{array}{l}\text { LUMO } \\
\left(\mathbf{E}_{\text {LUMO }}\right)\end{array}$ & $\begin{array}{l}\text { HOMO-LUMO } \\
\text { GAP }\end{array}$ & $\begin{array}{c}\text { Ionization } \\
\text { potential (I) }\end{array}$ & $\begin{array}{c}\text { Electron } \\
\text { Affinity (A) }\end{array}$ & $\begin{array}{c}\text { Global } \\
\text { Hardness } \\
(\eta)\end{array}$ & $\begin{array}{c}\text { Ch. } \\
\text { Potential } \\
(\mu)\end{array}$ & $\begin{array}{l}\text { Electrophilicity } \\
\qquad(\chi)\end{array}$ \\
\hline 1 & 1.07 & -0.326 & -0.2443 & 0.08162 & 0.326 & 0.24438 & 0.04081 & 0.28519 & 0.996487823 \\
\hline 2 & 5.97 & -0.3306 & -0.2275 & 0.1031 & 0.3306 & 0.2275 & 0.05155 & 0.27905 & 0.755275485 \\
\hline 3 & 2.77 & -0.3197 & -0.0471 & 0.27261 & 0.31976 & 0.04715 & 0.136305 & 0.183455 & 0.123457456 \\
\hline 4 & 2.68 & -0.3235 & -0.0450 & 0.27852 & 0.32354 & 0.04502 & 0.13926 & 0.18428 & 0.121927037 \\
\hline 5 & 4.18 & -0.3160 & -0.0383 & 0.27768 & 0.31601 & 0.03833 & 0.13884 & 0.17717 & 0.113040942 \\
\hline 6 & 3.15 & -0.3254 & -0.0486 & 0.27679 & 0.32545 & 0.04866 & 0.138395 & 0.187055 & 0.126411984 \\
\hline 7 & 6.44 & -0.3234 & -0.0351 & 0.28833 & 0.32343 & 0.0351 & 0.144165 & 0.179265 & 0.111455416 \\
\hline 8 & 4.91 & -0.3254 & -0.2043 & 0.12106 & 0.32542 & 0.20436 & 0.06053 & 0.26489 & 0.579602776 \\
\hline 9 & 7.83 & -0.2739 & -0.2342 & 0.03973 & 0.27393 & 0.2342 & 0.019865 & 0.254065 & 1.624692279 \\
\hline 10 & 7.04 & -0.3322 & -0.2248 & 0.10742 & 0.33229 & 0.22487 & 0.05371 & 0.27858 & 0.722461519 \\
\hline 11 & 5.15 & -0.3189 & -0.0387 & 0.28028 & 0.31898 & 0.0387 & 0.14014 & 0.17884 & 0.114113549 \\
\hline 12 & 9.28 & -0.2677 & -0.1722 & 0.09552 & 0.26776 & 0.17224 & 0.04776 & 0.22 & 0.506700168 \\
\hline 13 & 10.59 & -0.3314 & -0.1400 & 0.19139 & 0.33141 & 0.14002 & 0.095695 & 0.235715 & 0.290305456 \\
\hline
\end{tabular}

Studies relating the molecular properties of the compounds and their KDR inhibitory activity supported this hypothesis, as the descriptor dipole moment and SLogP show the higher contribution to the activity against KDR in the QSAR model, where contribution of electrophilicity is more significant than other descriptors. The higher LUMO energy is correlated with higher electron affinity. Henceforth, it concludes that increasing of electron affinity can increase the KDR inhibition activity. It is remarkable to note that increase in global hardness of the molecule leads to increase in stability and decrease in reactivity of the species (Ayers and Parr, 2000). From eq. (3), it also confirms that hardness of these molecules influence their inhibitory activity. However as a whole, this result is anticipated as the hydrophobicity and lipophilicity of the chemical compounds mainly govern their biological actions at cellular and molecular levels.

\section{CONCLUSION}

The current study is designed to reveal the therapeutic potentiality of some selected N-4-chlorophenylnaphthamide derivatives regarding the perspectives in tyrosine kinase inhibitor. In collateral, our findings agree with the existing evidence with concluding a better pharmacokinetics profile. The results of QSAR and ADME/T studies are validated each other and the developed mathematical models could provide insight into the structural requirements for the synthesis of new potential chemical structure having a better KDR inhibition activity.

\section{Financial support and sponsorship: Nil.}

Conflict of Interests: There are no conflicts of interest.

\section{REFERENCES}

Adjei AA. Novel small-molecule inhibitors of the vascular endothelial growth factor receptor. Clinical lung cancer. 2007;8:S74-S8.

Aiello LP, Avery RL, Arrigg PG, Keyt BA, Jampel HD, Shah ST, et al. Vascular endothelial growth factor in ocular fluid of patients with diabetic retinopathy and other retinal disorders. New England Journal of Medicine. 1994;331(22):1480-7.
Ayers PW, Parr RG. Variational principles for describing chemical reactions: the Fukui function and chemical hardness revisited. Journal of the American Chemical Society. 2000;122(9):2010-8.

Beebe JS, Jani JP, Knauth E, Goodwin P, Higdon C, Rossi AM, et al. Pharmacological characterization of CP-547,632, a novel vascular endothelial growth factor receptor-2 tyrosine kinase inhibitor for cancer therapy. Cancer research. 2003;63(21):7301-9.

Bundy JG, Morriss AW, Durham DG, Campbell CD, Paton GI. Development of QSARs to investigate the bacterial toxicity and biotransformation potential of aromatic heterocylic compounds. Chemosphere. 2001;42(8):885-92.

Cardones AR, Banez LL. VEGF inhibitors in cancer therapy. Current pharmaceutical design. 2006;12(3):387-94.

Cherrington JM, Strawn LM, Shawver LK. New paradigms for the treatment of cancer: the role of anti-angiogenesis agents. Advances in cancer research. 2000;79:1-38.

Choudhary D, Gupta GK, Khokra SL. Structure based designing and ADME-T studies of butenolide derivatives as potential agents against receptor ICAM-1: A drug target for cerebral malaria. Journal of Computational Science. 2015;10:156-65.

D'Adamo DR, Anderson SE, Albritton K, Yamada J, Riedel E, Scheu K, et al. Phase II study of doxorubicin and bevacizumab for patients with metastatic soft-tissue sarcomas. Journal of Clinical Oncology. 2005;23(28):7135-42.

Dash R, Hosen S, Karim M, Kabir MSH, Hossain MM, Junaid $\mathrm{M}$, et al. In silico analysis of indole-3-carbinol and its metabolite DIM as EGFR tyrosine kinase inhibitors in platinum resistant ovarian cancer vis a vis ADME/T property analysis. J App Pharm Sci, 2015; 5 (11): 073-078.

Detmar M. The role of VEGF and thrombospondins in skin angiogenesis. Journal of dermatological science. 2000;24:S78S84.

Egan WJ, Merz KM, Baldwin JJ. Prediction of drug absorption using multivariate statistics. Journal of medicinal chemistry. 2000;43(21):3867-77

Fava GA. Affective disorders and endocrine disease: new insights from psychosomatic studies. Psychosomatics. 1994;35(4):341-53.

Ferrara N. VEGF and the quest for tumour angiogenesis factors. Nature Reviews Cancer. 2002;2(10):795-803.

Folkman J. Anti-angiogenesis: new concept for therapy of solid tumors. Annals of surgery. 1972;175(3):409.

Geladi P, Kowalski BR. Partial least-squares regression: a tutorial. Analytica chimica acta. 1986;185:1-17.

Gingrich DE, Reddy DR, Iqbal MA, Singh J, Aimone LD, Angeles TS, et al. A new class of potent vascular endothelial growth factor receptor tyrosine kinase inhibitors: structure-activity relationships for a series of 9-alkoxymethyl-12-(3-hydroxypropyl) indeno [2, 1-a] pyrrolo [3, 4-c] carbazole-5-ones and the identification of CEP-5214 and its dimethylglycine ester prodrug clinical candidate CEP-7055. Journal of medicinal chemistry. 2003;46(25):5375-88. 
Halgren TA. Merck molecular force field. I. Basis, form, scope, parameterization, and performance of MMFF94. Journal of computational chemistry. 1996;17(5-6):490-519.

Harmange J-C, Weiss MM, Germain J, Polverino AJ, Borg G, Bready J, et al. Naphthamides as Novel and Potent Vascular Endothelial Growth Factor Receptor Tyrosine Kinase Inhibitors: Design, Synthesis, and Evaluation. Journal of medicinal chemistry. 2008;51(6):1649-67.

Helland IS. On the structure of partial least squares regression. Communications in statistics-Simulation and Computation. 1988; 17(2): 581-607.

Hennequin LF, Stokes ES, Thomas AP, Johnstone C, Plé PA, Ogilvie DJ, et al. Novel 4-anilinoquinazolines with C-7 basic side chains: design and structure activity relationship of a series of potent, orally active, VEGF receptor tyrosine kinase inhibitors. Journal of medicinal chemistry. 2002; 45(6): 1300-12.

Hughes LD, Palmer DS, Nigsch F, Mitchell JB. Why are some properties more difficult to predict than others? A study of QSPR models of solubility, melting point, and Log P. Journal of chemical information and modeling. 2008; 48(1): 220-32.

Kapur S, Shusterman A, Verma RP, Hansch C, Selassie CD. Toxicology of benzyl alcohols: a QSAR analysis. Chemosphere. 2000;41(10):1643-9.

Kilari S, Remadevi I, Zhao B, Pan J, Miao R, Ramchandran R, et al. Endothelial Cell-specific Chemotaxis Receptor (ECSCR) Enhances Vascular Endothelial Growth Factor (VEGF) Receptor-2/Kinase Insert Domain Receptor (KDR) Activation and Promotes Proteolysis of Internalized KDR. The Journal of Biological Chemistry. 2013; 288(15): 10265-74.

Li M-y, Lv Y-c, Tong L-j, Peng T, Qu R, Zhang T, et al. DW10075, a novel selective and small-molecule inhibitor of VEGFR, exhibits antitumor activities both in vitro and in vivo. Acta Pharmacologica Sinica. 2016;37(3):398-407.

Liotta LA, Steeg PS, Stetler-Stevenson WG. Cancer metastasis and angiogenesis: an imbalance of positive and negative regulation. Cell. 1991;64(2):327-36.

Quentmeier H, Eberth S, Romani J, Weich HA, Zaborski M, Drexler HG. DNA methylation regulates expression of VEGF-R2 (KDR) and VEGF-R3 (FLT4). BMC Cancer. 2012;12:19-.

Ray S, Madrid PB, Catz P, LeValley SE, Furniss MJ, Rausch $\mathrm{LL}$, et al. Development of a new generation of 4-aminoquinoline antimalarial compounds using predictive pharmacokinetic and toxicology models. Journal of medicinal chemistry. 2010;53(9):3685-95.
Ren S, Frymier PD. Estimating the toxicities of organic chemicals to bioluminescent bacteria and activated sludge. Water research. 2002;36(17):4406-14.

Ruggeri B, Singh J, Gingrich D, Angeles T, Albom M, Chang $\mathrm{H}$, et al. CEP-7055 A Novel, Orally Active Pan Inhibitor of Vascular Endothelial Growth Factor Receptor Tyrosine Kinases with Potent Antiangiogenic Activity and Antitumor Efficacy in Preclinical Models. Cancer research. 2003;63(18):5978-91.

Ryan A, Wedge S. ZD6474-a novel inhibitor of VEGFR and EGFR tyrosine kinase activity. British journal of cancer. 2005;92:S6-S13.

Sun L, Liang C, Shirazian S, Zhou Y, Miller T, Cui J, et al. Discovery of 5-[5-fluoro-2-oxo-1, 2-dihydroindol-(3 Z)-ylidenemethyl]-2, 4-dimethyl-1 H-pyrrole-3-carboxylic acid (2-diethylaminoethyl) amide, a novel tyrosine kinase inhibitor targeting vascular endothelial and plateletderived growth factor receptor tyrosine kinase. Journal of medicinal chemistry. 2003;46(7):1116-9.

Terman BI, Dougher-Vermazen M, Carrion ME, Dimitrov D, Armellino DC, Gospodarowicz D, et al. Identification of the KDR tyrosine kinase as a receptor for vascular endothelial cell growth factor. Biochemical and biophysical research communications. 1992; 187(3): 1579-86.

Thomas AL, Morgan B, Drevs J, Unger C, Wiedenmann B, Vanhoefer U, et al., editors. Vascular endothelial growth factor receptor tyrosine kinase inhibitors: PTK787/ZK 222584. Seminars in oncology; 2003: Elsevier.

Walsh D, Haywood L. Angiogenesis: a therapeutic target in arthritis. Current opinion in investigational drugs (London, England: 2000). 2001;2(8):1054-63.

Worgan AD, Dearden JC, Edwards R, Netzeva TI, Cronin MT. Evaluation of a Novel Short-Term Algal Toxicity Assay by the Development of QSARs and Inter-Species Relationships for Narcotic Chemicals. QSAR \& Combinatorial Science. 2003;22(2):204-9.

\section{How to cite this article:}

Hosen SMZ, Dash R, Khatun M, Akter R, Bhuiyan MHR, Karim MR, Mouri NJ, Ahmed F, Islam KS, Afrin S. In silico ADME/T and 3D QSAR analysis of KDR inhibitors. J App Pharm Sci, 2017; 7 (01): 120-128 